\title{
Les vergers dans les opérations d'aménagement global en Lorraine
}

The contribution of orchards to country planing development in Lorraine

Die Obstgärten in den globalen Raumordnungsverfahren in Lothringen

\section{Aurélie Rin et Jean-Pierre Husson}

\section{(2) OpenEdition}

\section{Journals}

Édition électronique

URL : http://journals.openedition.org/rge/2610

DOI : $10.4000 /$ rge. 2610

ISSN : $2108-6478$

Éditeur

Association des géographes de l'Est

Édition imprimée

Date de publication : 1 juin 2002

ISSN : 0035-3213

\section{Référence électronique}

Aurélie Rin et Jean-Pierre Husson, « Les vergers dans les opérations d'aménagement global en

Lorraine », Revue Géographique de l'Est [En ligne], vol. 42 / 3 | 2002, mis en ligne le 14 décembre 2010, consulté le 08 septembre 2020. URL : http://journals.openedition.org/rge/2610 ; DOI : https://doi.org/ 10.4000/rge.2610

Ce document a été généré automatiquement le 8 septembre 2020

Tous droits réservés 


\title{
Les vergers dans les opérations d'aménagement global en Lorraine
}

\author{
The contribution of orchards to country planing development in Lorraine \\ Die Obstgärten in den globalen Raumordnungsverfahren in Lothringen
}

\author{
Aurélie Rin et Jean-Pierre Husson
}

1 Les vergers lorrains, servant d'écrin aux villages, sont placés entre déclin, enfrichement, abandon lié à l'indivision parcellaire ou encore réattribution, mise en scène des paysages. Leur histoire résulte des scénarios liés à l'alternance avec les vignes. Les vergers longtemps malmenés, oubliés, sont aujourd'hui réinvestis, partiellement convoités, parfois placés au cœur de nouveaux enjeux d'image et de médiation de territoire. Il s'agit donc de terroirs en devenir, champ de projets et d'expériences intéressants, originaux, parfois inédits, réintroduisant une approche globale de compréhension, d'aménagement et de lecture paysagère des sites (Croix, 1998 ; Berger, 2001).

2 Plus spécialement, en Lorraine, c'est le mirabellier à haute tige qui fut l'arbre emblématique des vergers, servant de décor familier aux villages groupés (Husson, 2001). Partout, la dynamique vigne-verger fut complexe, exercée en négatif de l'autre culture ou encore par rapport à la forêt. Les vergers sont des terroirs de typicité. Leurs arbres donnent les coloris aux paysages des meix, harmonisent et fondent l'habitat dans un écrin parfois " artialisé » (expression empruntée à Montaigne qui voit dans le paysage le décor de l'œuvre peinte) par les peintres et graveurs. Dans le finage de côte, objet géographique majeur, les vergers sont souvent à l'interface des trois espaces : le bâti, la SAU désormais organisée en openfield mosaïque et les lisières forestières stables ou conquérantes par enfrichement continu ou ponctuel (ourlet de friches s'effilochant).

Vieux vergers maltraités par le temps, vergers restés fonctionnels et nouveaux vergers ont migré sur les versants. Sur les premiers cadastres, les vergers occupent en général le haut des pentes, placés entre lisière et vigne, dessinant une gradation du couvert (Mathy, 1998). Ils profitent au mieux des réductions des chocs thermiques liés à leur situation privilégiée. A la fin du XIX ${ }^{e}$ siècle, dans un contexte de déclin tardif et inégal 
de la vigne, le verger a opéré un glissement latéral, étant remplacé par la fructicée dans les parties amont pentues, caillouteuses, difficiles à travailler. En lutte avec l'essor du bâti, les vergers actuels ont tendance à poursuivre leur descente latérale, les vergers industriels occupant les bas des versants, sur des sites de pentes faibles compatibles avec la mécanisation de l'activité fruitière. Les vergers actuels affichent une cohérence multifonctionnelle définie par une grande inventivité pour concevoir, pour doser la cohabitation des fonctions de production labellisées, de préservation d'image (Hervieu et Viard, 1996), de biodiversité, de nouvelle cohérence à apporter aux sections bâties des villages.

4 L'exposé replace les vergers dans le cadre élargi de l'aménagement rural global. Désormais, les vergers apparaissent tout à la fois comme des espaces patrimoniaux et le support à une production originale identifiée régionalement. Sur le terrain, les diverses générations de vergers coexistent et sont très souvent soumises à la double pression de la friche et de l'étalement urbain (zone susceptible de future urbanisation (Leclaire 2002). Leur pérennité s'inscrit dans une problématique de cohérence paysagère. Celle-ci impose de remédier à l'émiettement parcellaire et de fédérer les propriétaires autour du paysage avec la reconnaissance productive des vergers à dégager des friches, éventuellement à faire renaître par des restructurations foncières. Ainsi, le projet en cours à Villey-Saint-Étienne concerne 767 parcelles du versant de la Moselle, majoritairement imbriquées les unes dans les autres, envahies par un fouillis de friches qui évoluent vers le taillis. Le remaniement de ce micro-parcellaire doit permettre à la commune de faire de nouvelles acquisitions foncières, dont un des objectifs concerne la plantation de nouveaux vergers à vocation pédagogique. La seconde partie du texte illustre le propos tenu par l'énoncé, hélas trop rare, des cas concrets de reconnaissance des vergers.

\section{Les vergers : un terroir pour une nouvelle écologie du paysage}

\section{A. Un patrimoine naturel à préserver en espace rural}

\section{Les vergers font partie du patrimoine des finages des systèmes de côte}

5 L'authenticité et la richesse des milieux naturels confèrent aux paysages élaborés sur les terroirs complémentaires des systèmes de côte (Jacquin et al., 1994) une forte valeur patrimoniale. Les nouvelles lois promulguées en faveur de la protection de l'environnement et des paysages renforcent cette idée, marquée par une prise de conscience récente et croissante de la nécessité de préserver, non seulement les paysages remarquables, mais également les paysages sans originalité, reconnus comme cadres de vie et comme terroirs de qualité. Les paysages ruraux, hérités du passé et façonnés par le travail des hommes, renvoient aux sociétés agricoles qui se sont succédées. Les vergers sont présents sur la section village et sur les côtes. Ils recèlent de nombreuses richesses qui méritent d'être mises en valeur. Située principalement dans le meix, en arrière des habitations, la ceinture arborée est un témoin de l'organisation traditionnelle des villages sous les côtes. Elle constitue un cadre paysager d'une indéniable valeur (figure 1). 
Figure 1 : Le meix de Bauzemont

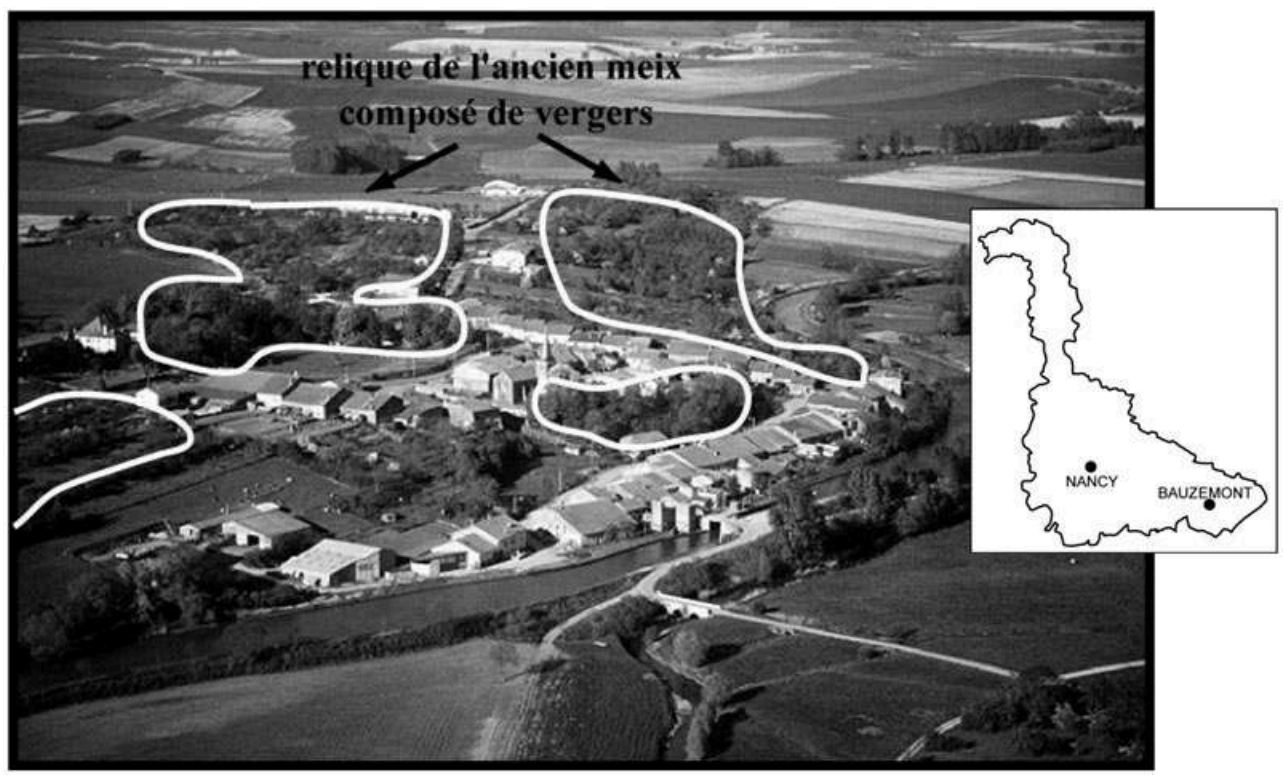

Les communes lorraines sont traditionnellement entourées par un meix arboricole composé de jardins et de vergers. Actuellement, cette ceinture verte est relique, coupée par des parcelles de terrains rendus constructibles.

cliché Humbert, CERPA, Bauzemont 94-322

6 Actuellement, les vergers connaissent ou subissent des situations évolutives très variées en fonction des intérêts qu'ils suscitent, de la topographie (figure 2), de l'intérêt des propriétaires et de l'implication des municipalités, et enfin de la qualité des restructurations foncières si celles-ci existent et intègrent les espaces concernés par les vergers. D'autre part, le souci de rétablir une récolte variée et de qualité régulière, si possible peu affectée par des coups de gels tardifs, s'impose. Il conduit à diversifier les productions (pommiers et poiriers sur les côtes de Meuse, dans le pays de Bayon, sur les versants bien exposés du Grand Couronné, cerisiers autour de la colline de Sion, quetschiers ailleurs, ...). 
Figure 2 : Emplacement des vergers dans les communes en fonction de la topographie locale.

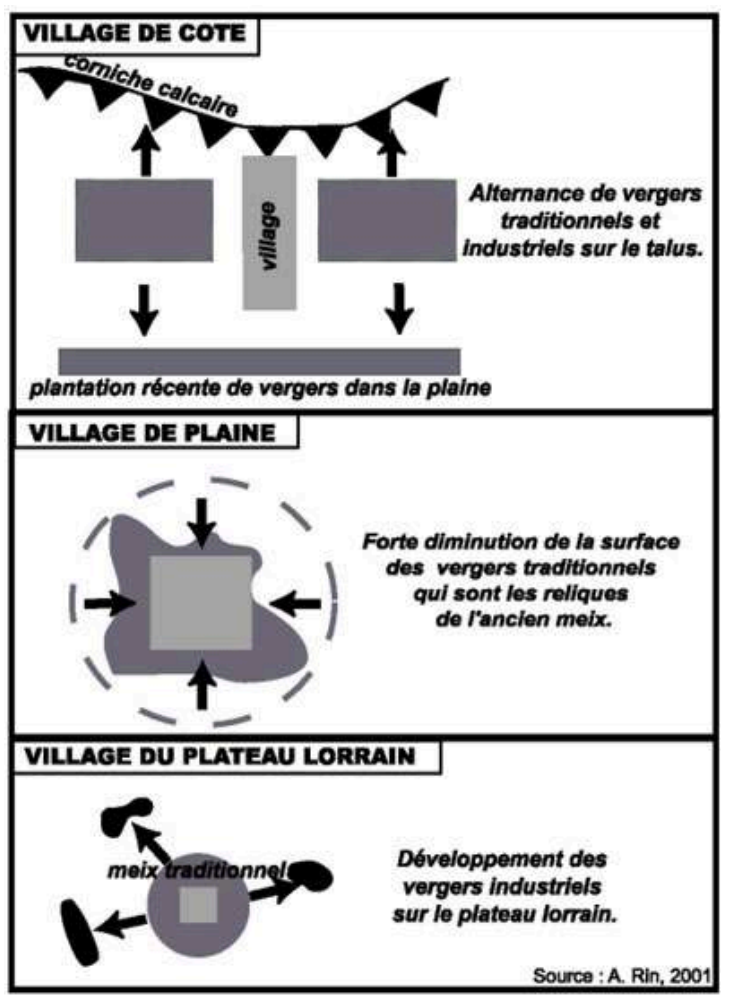

2. Le développement de la labellisation, un début de reconnaissance des vergers.

7 Après une phase de déclin prolongé, longtemps jugée irréversible, le début des années quatre-vingts apparait comme une période de rémission ou d'arrêt du recul et de la dégradation des vergers. L'effort effectué en faveur du replant de jeunes arbres améliore, rééquilibre la pyramide des âges des vergers. De nouvelles réflexions qualitatives, environnementales et paysagères, apparaissent progressivement. Elles replacent les vergers dans les problématiques qui deviennent ultérieurement celles du développement rural durable. La mise en place de labels, de certifications et l'émergence de plusieurs associations montrent que les vergers retrouvent de leur intérêt au sein d'un jeu d'actions redéfini, élargi, sorti d'une logique agricole exclusive. En 1994, deux sites lorrains ont obtenu le label «Reconquête des paysages » décerné par le ministère de l'Environnement pour la sauvegarde et la préservation des paysages ruraux. Il s'agit du Bayonnais et de la colline de Sion. Le label rouge a pour objectif de reconnaître des sites régionaux remarquables pour leur spécificité paysagère mais aussi pour les activités arboricoles qui soutiennent ces terroirs. «(...) Ils attestent qu'une denrée alimentaire possède un ensemble distinct de qualités et caractéristiques spécifiques préalablement fixées dans un cahier des charges et établissant un niveau de qualité supérieure si elle est enregistrée comme Indication Géographique Protégée. Ce produit doit se distinguer des produits similaires de l'espèce habituellement commercialisée, notamment par ses conditions particulières de production ou de fabrication et, le cas échéant, par son origine géographique. Seuls des producteurs ou des transformateurs organisés en groupement, quelle qu'en soit la forme juridique, sont habilités à la demande de délivrance d'un label. Ces labels agricoles ne peuvent êtres utilisés que s'ils ont fait l'objet d'une homologation par arrêté interministériel (Article 1). »(extrait du texte relatif à l'obtention du label « reconquête des paysages »). 
Labelliser les paysages permet ainsi, à travers une reconnaissance publique, de soutenir les activités spécifiques et d'intéresser les collectivités locales à la gestion de leurs territoires. Cette labellisation s'inscrit dans une politique de développement local, de projet fédérateur. Après presque un demi-siècle de déclin, d'évolution soulignée par de fortes disparités, des dysfonctionnements, des formes de renaissance, les vergers entrent en force dans les approches globales, économiques, mais aussi esthétiques. L'association "Mirabelles de Lorraine ", créée en 1995, regroupe des arboriculteurs et des entreprises passionnés par la mirabelle et qui veulent respecter des règles strictes de production. Depuis 1996, elle est également détentrice de l'Indication Géographique Protégée. L'appellation et la marque « Mirabelle de Lorraine » apportent la garantie aux consommateurs d'un produit fin, bon, traditionnel, respectant l'environnement. Un cahier des charges impose le respect de règles de production très strictes. Il garantit la récolte et le conditionnement des fruits, une teneur en sucre minimale, un produit identifié du producteur au consommateur, avec entre autre une taille de fruits toujours supérieure à 22 millimètres. Les vergers bénéficient depuis quelques temps des mesures agri-environnementales (Kervalla, 1995). Mises en place depuis les années quatre-vingtdix, elles sont encore trop peu appliquées. Etablies sous forme de contrat entre l'agriculteur et l'Etat, elles permettent d'une part, de développer des pratiques agricoles compatibles avec la préservation des biotopes remarquables, et d'autre part, de maintenir des pratiques agricoles limitant les menaces pouvant peser sur la qualité des paysages et sur la diversité biologique. Ces mesures permettent ainsi de réhabiliter les parcelles arboricoles en prenant en compte la problématique paysagère dans les vergers. Elles ont été relayées par les CTE (contrats territoriaux d'exploitation) avant que ces contrats ne soient suspendus.

\section{Les collectivités locales se mobilisent}

8 Les vergers de fruitiers constituent une richesse paysagère et patrimoniale indéniable qu'il faut préserver. Pour cela, les collectivités territoriales et locales se mobilisent et les intègrent dans leur politique d'aménagement rural. La question des vergers contribue à faire émerger l'image de terroirs équilibrés, esthétiques, voire idéalisés. La mise en place de plusieurs initiatives confirme cette volonté de pérenniser les vergers tant pour leur caractère paysager traditionnel que pour leur production fruitière, même si celle-ci n'est qu'un revenu d'appoint irrégulier. Les actions conduites peuvent émaner du département (politique de relance fruitière mise en place par le conseil général de Moselle), d'une commune (problématique des vergers intégrée dans le plan paysage de Leyr, Meurthe-et-Moselle), des parcs naturels régionaux (création des vergers-conservatoires par le Parc Naturel Régional de Lorraine à Hannonville en Meuse), de structures intercommunales, du centre INRA d'Ars-Laquenexy, diffuseur de nouvelles variétés utilisées (figure 3). 
Figure 3 : les vergers sont à l'interface de multiples enjeux

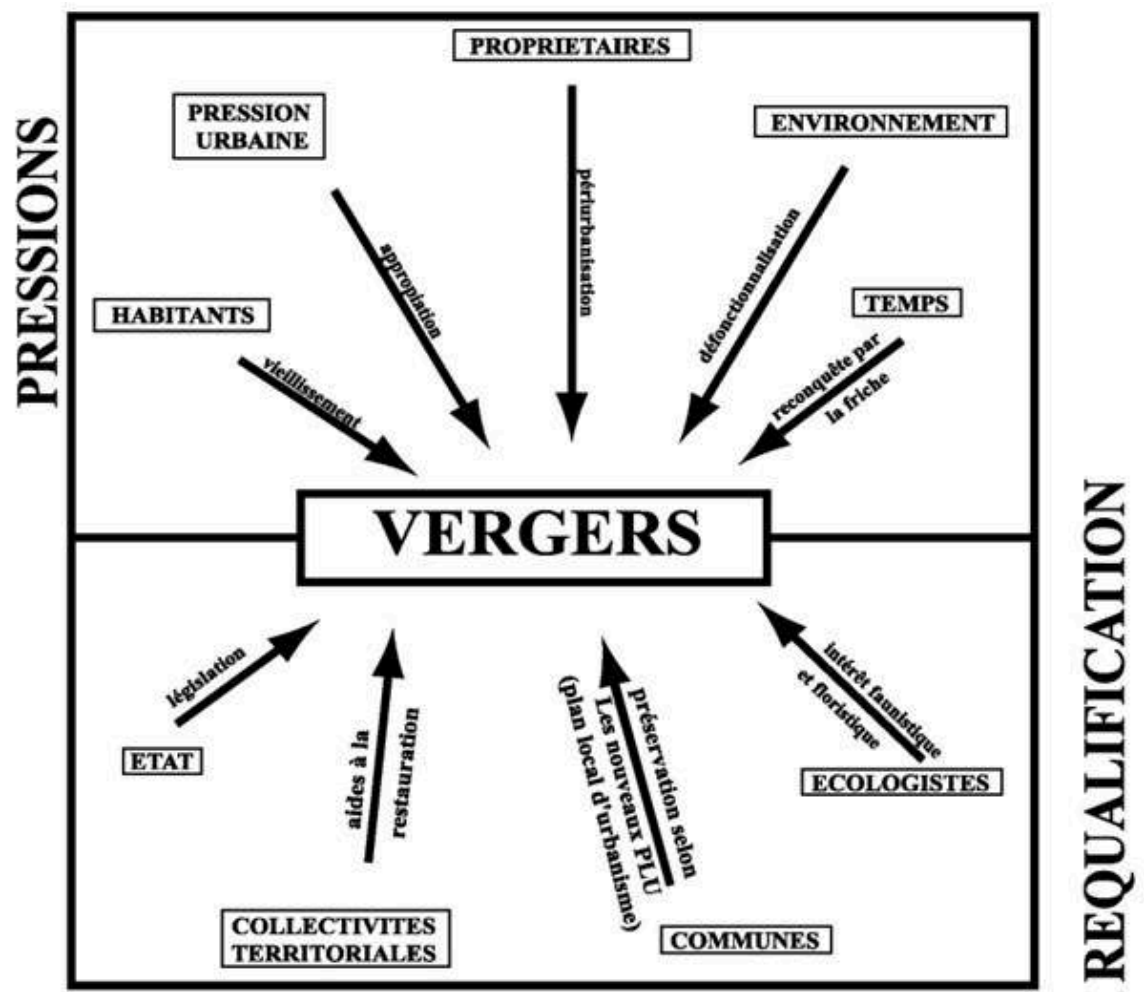

Actuellement, la place des vergers est intégrée dans les nouvelles préoccupations de développement rural durable. La réalité n'est pas toujours en adéquation avec les ambitions de pérennité avancées. Les vergers sont soumis à de nombreuses pressions qui expliquent l'abandon et l'enfrichement de beaucoup de parcelles.

Dans le cadre d'une nouvelle politique en faveur des vergers s'est illustrée la communauté de communes du Saintois. Elle a mené avec succès des actions de rénovation et de renouvellement des arbres fruitiers abîmés par la tempête du 26 décembre 1999. Créée en janvier 2001, l'association "Vignes et vergers du Saintois " œuvre pour encourager le maintien des vergers, transmet un savoir-faire aux jeunes générations en matière de taille, de greffe, de plantation et de valorisation de la production fruitière.

Depuis 1981, L'AREFE (Association Régionale pour l'Exploitation Fruitière Est) regroupe des producteurs agricoles qui souhaitent maittriser leur production et conserver les variétés traditionnelles de mirabelles. Actuellement, cette structure dispose de 21 hectares de vergers à Vigneulles-lès-Hattonchatel (Meuse) où elle conserve des variétés anciennes de mirabelliers, de cerisiers, de pêchers, de pommiers et d'abricotiers. L'AREFE fait partie d'un réseau national de 25 stations d'expérimentation de fruits et légumes. Le CTIFL (Centre Technique Interprofessionnel des Fruits et Légumes) en coordonne les programmes. En rendant les producteurs attentifs à l'aspect technique de l'arboriculture, cette association contribue à la promotion de la culture de la mirabelle. L'AREFE mène plusieurs travaux qui peuvent être diffusés dans d'autres régions grâce au relais du CTIFL Parmi ses actions, on peut noter la mise en place et la conduite des vergers, le choix de nouveaux porte-greffes, la lutte contre les maladies, la définition des critères de qualité du fruit. 
11 Les collectivités locales impliquées peuvent bénéficier de la mise en place d'une OPAV L'Opération programmée d'Amélioration des Vergers a pour objectif d'arrêter le recul spatial et qualitatif des vergers familiaux. Il s'agit d'une création de "Meuse Nature Environnement ", association reconnue d'utilité publique, fondée en 1964. Elle mène une réflexion et des actions sur les vergers afin de contrer efficacement le mouvement de déprise qui était parfois un abandon total des vergers. L'OPAV se place désormais à la convergence de nouvelles attentes exprimées par une gamme d'acteurs ruraux différente de celle du passé. L'expérience des OPAV a été initialement limitée au département de la Meuse. Récemment, elle a été étendue à toute la Lorraine. Un réseau régional Vergers réunit et coordonne l'ensemble des maîtres d'œuvre des OPAV lorraines. Ces actions sont intégrées dans les politiques des paysages conduites par la DIREN (Direction Régionale de l'Environnement).

\section{B. Le recul spatial et l'enfrichement des vergers sont-ils arrêtés ?}

\section{L'abandon des parcelles de vergers.}

12 L'abandon des vergers est un phénomène ancien, commencé dès les années trente. L'ampleur de ce phénomène s'est accrue depuis une trentaine d'années, révélateur de pratiques et de comportements nouveaux qui commencent à s'inverser, à être critiqués. L'abandon des parcelles de vergers conduit à l'enfrichement de la majorité des terroirs de côte, à la dilution de la lisière forestière conquérante grâce à la multiplication des essences héliophiles (Bonnefont, 1974) (figure 4). 
Figure 4 : Le front de côte de la commune de Lucey (Côtes de Toul)

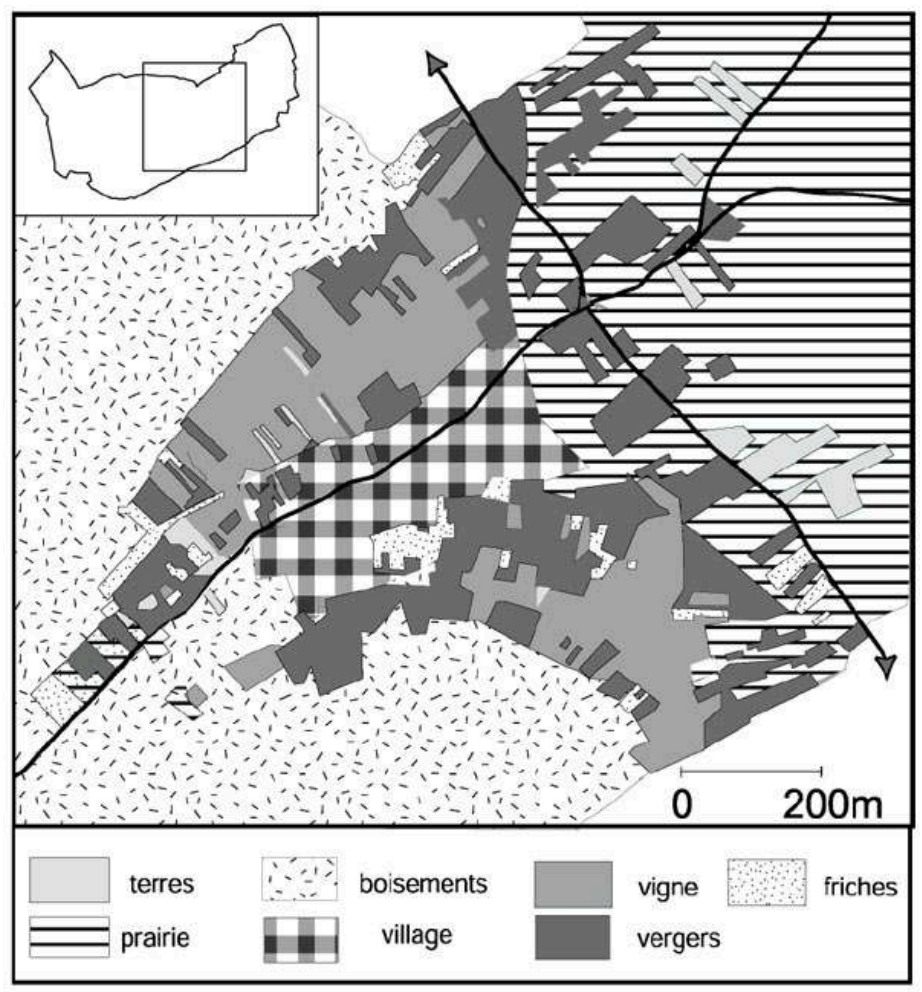

Comme dans tous les terroirs de côte, la commune de Lucey est marquée par un enfrichement des parcelles de vergers et une avancée de la lisière forestière. Dans le secteur des Côtes de Toul, de nombreux vergers abandonnés, délaissés par leurs propriétaires, sont transformés en terrains à bâtir ou regagnés par le vignoble.

d'après A. Rin, 2001

Le recul spatial des vergers est inscrit sur plusieurs décennies et s'explique par la conjugaison de données ayant créé une synergie négative : parcellaire très émietté, propriétaires vieillissants, désintéressés de leurs biens, absents. Actuellement, la conservation des parcelles dépend, dans la majorité des cas, de la volonté de préservation et de l'attachement affiché par le propriétaire, de son âge, de son implication. Beaucoup de propriétaires sont partis, géographiquement trop éloignés ou trop âgés pour assurer l'entretien de leur bien. Après plusieurs successions, les nouveaux propriétaires ont $\mathrm{pu}$ oublier l'existence de leurs parcelles. Faute de rendement suffisant dans le recouvrement de l'impôt, le ministère des Finances abandonne la recherche concernant l'assiette fiscale relative aux microparcelles des propriétaires non résidents. Le foncier est souvent figé car les propriétaires préfèrent délaisser leurs terres plutôt que de les vendre, avec des frais notariés et des démarches à mener sans rapport avec le prix réel du bien à céder. Le déblocage d'une situation foncière figée est délicat à lever, complexifié par les nombreux cas d'indivision et par les réflexes de suspicion à attendre d'un éventuel changement d'affectation de PLU (plan local urbain) en zone où s'effectuent des constructions. Les actuels vergers en abandon s'inscrivent partiellement dans une logique de friche d'attente spéculative.

Le désengagement des propriétaires et son corollaire, la friche, expliquent les différentes étapes constatées, voire annoncées dans les processus d'évolution des vergers. Tous les cas de figure et tous les stades d'évolution se rencontrent sur une même commune. Les arbres âgés, sénescents, défigurés, sont nombreux. Ils 
s'individualisent par leur stature et leur port, résultat d'une taille en haute tige. Lesvergers en cours d'enfrichement créent des paysages flous, évolutifs. Ils fossilisent un parcellaire très émietté relié par un lacis de petits chemins qui peuvent perdre leur fonctionnalité s'ils sont peu fréquentés. Ces paysages flous avancent par ourlets successifs, présentant tous les stades de reconquête. Les vergers sont d'abord envahis par les ronces qui se transforment en fourrés impénétrables et cohabitent avec des arbres surannés, souvent cassés. Cette reconquête forestière, longtemps perçue de manière négative, est actuellement reconnue pour la qualité de son habitat ou le rôle de réserve foncière qu'elle peut jouer dans le cadre des stratégies édictées par l'Europe Verte (dont la récente mise en place des mesures agri-environnementales). En outre, la tempête du 26 décembre 1999 a brusquement accéléré l'évolution des vergers déjà fragilisés, causant de nombreux dégâts parfois irréversibles, en particulier sur les versants exposés aux flux venus d'ouest. Les arbres ont été arrachés, déracinés ou simplement vrillés sur eux-mêmes, le vent brisant leurs racines ou fissurant leurs écorces. Après le passage de Lothar, beaucoup d'espèces anciennes plus savoureuses que les nouvelles ont disparu. Seuls les vergers professionnels, plantés récemment, taillés en gobelet, donnant peu de prise au vent, ont résisté avec seulement $1 \%$ de perte en Meurthe-et-Moselle.

\section{Les remembrements}

15 Depuis moins d'une dizaine d'années, les opérations d'aménagement foncier ont largement évolué (Marochini, 1999), passant d'une recherche presque exclusive de gains de productivité par la simplification du parcellaire, à une démarche plutôt qualitative et globale incluant la préservation des paysages. Aujourd'hui, l'opération de remembrement commence à intégrer une réflexion particulière sur le parcellaire arboricole. Cependant, en pratique, il faut reconnaître que certaines communes éludent encore complètement cette problématique alors que d'autres mènent une réflexion approfondie. En effet, l'exclusion des vergers du périmètre de remembrement reste fréquente. Ce choix est une solution de facilité pour contourner les problèmes posés par leur devenir. Même si la préservation des vergers permet de dégager un certain consensus, le passage à l'acte, autrement dit le remembrement de vergers, reste rare. Sa réalisation lève de nombreux conflits qui sont davantage d'ordre affectif que relevant de réels enjeux économiques. La population locale peut faire bloc et refuser l'aménagement foncier. Les propriétaires sont souvent réticents à toute modification du parcellaire arboricole prétextant leur attachement à la terre. Pourtant, l'inclusion des vergers dans le périmètre de remembrement permet de restructurer ce parcellaire extrêmement morcelé et enclavé, de créer de nouveaux chemins de desserte, de planter de nouveaux arbres.

\section{II. Étude de cas : la conception des vergers au service d'une approche globale rurbaine}

Cet article qui souligne les efforts prodigués, les changements entrepris, les projets portés, ne doit pas faire oublier que la majorité des surfaces des vergers lorrains reste malmenée, souffrante, abandonnée. Les études de cas présentées font encore exception. Leurs aboutissements doivent servir de base de réflexion à une requalification globale et plurifonctionnelle des territoires ruraux et périurbains concernés par la présence 
des vergers. Seule cette conception peut mobiliser une gamme ouverte d'acteurs et aboutir à un investissement supportable.

\section{A. Leyr (Meurthe-et-Moselle) : une intégration réussie des vergers dans un remembrement}

17 Dans la majorité des processus du remembrement, les vergers sont envisagés comme un problème secondaire. La commune de Leyr se démarque de ce choix. Elle peut être prise comme modèle de réflexion nouvelle à mettre en place. Leyr est située à 18 kilomètres au nord de Nancy. La commune s'inscrit désormais dans le périmètre défini par la loi SRU (loi relative à la solidarité et au renouvellement urbain du 13 décembre 2000). Ce cas particulier démontre que les vergers peuvent être au cœur des discussions et bloquer le déroulement d'un remembrement (ici pendant près de dix ans) lorsqu'ils ne sont pas appréhendés à leur juste valeur. A Leyr, les agriculteurs ont demandé le remembrement dès 1988. La commune n'avait jamais été remembrée. Cette refonte foncière devenait nécessaire au bon déroulement de l'activité agricole. Suite à de nombreux conflits générés par une action menée sans toutes les négociations suffisantes, la procédure a été abandonnée dès 1989 avant d'être reprise en 1994, puis en 1998. C'est seulement à cette date que commence réellement le remembrement. Contrairement aux tentatives précédentes, il fut décidé de consulter, d'informer amplement l'ensemble de la population locale et de se conformer à la politique décidée par le département. Un plan paysage a été réalisé en 1998 conjointement à la mise en place de la pré-étude d'aménagement foncier (1999). Il prend en compte les contraintes environnementales et énonce le souci de la préservation des paysages. Ce plan était particulièrement chargé de déterminer les actions à mener sur la couronne de vergers située dans l'ancien meix (figure 5), périmètre dégradé mais également lieu de toutes les ambitions spéculatives pour cette commune désormais toute proche du pôle logistique nord de Nancy (Champigneulles-Frouard). 
Figure 5 : Leyr, une couronne de vergers en cours de dégradation

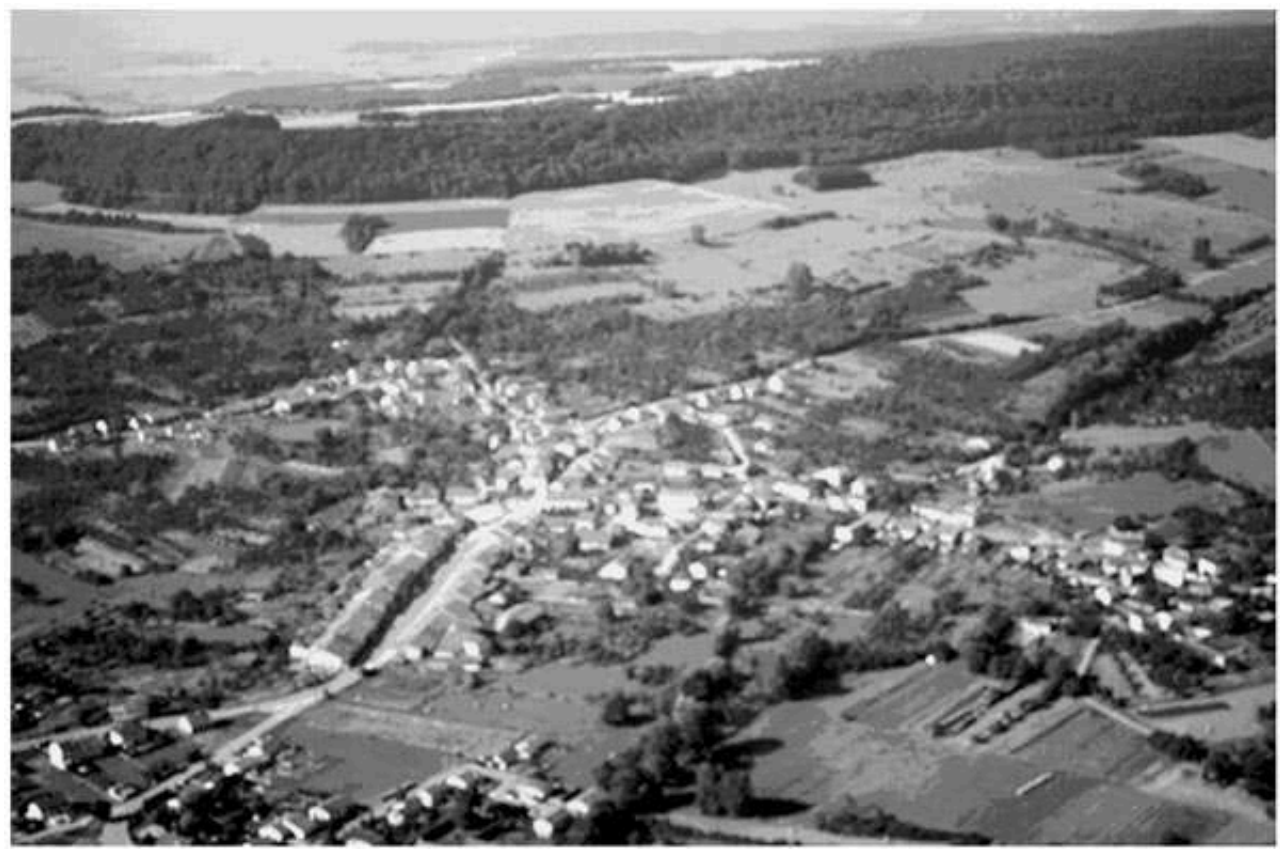

Les vergers situés en périphérie du noyau villageois sont plus ou moins bien entretenus et beaucoup d'entre eux sont actuellement abandonnés. La volonté des propriétaires de les maintenir a amené la municipalité à engager un plan paysage afın de restructurer ce parcellaire en cours de dégradation. Cliché : A. Humbert, CERPA, Leyr, 94-1106

Face à la complexité de la situation, le périmètre de remembrement a dû être remanié plusieurs fois afin de prendre en compte la spécificité des vergers, les contraintes engendrées par leur présence et les demandes formulées par les exploitants (figure 6). 
Figure 6 : Le périmètre de remembrement de la zone « verger »

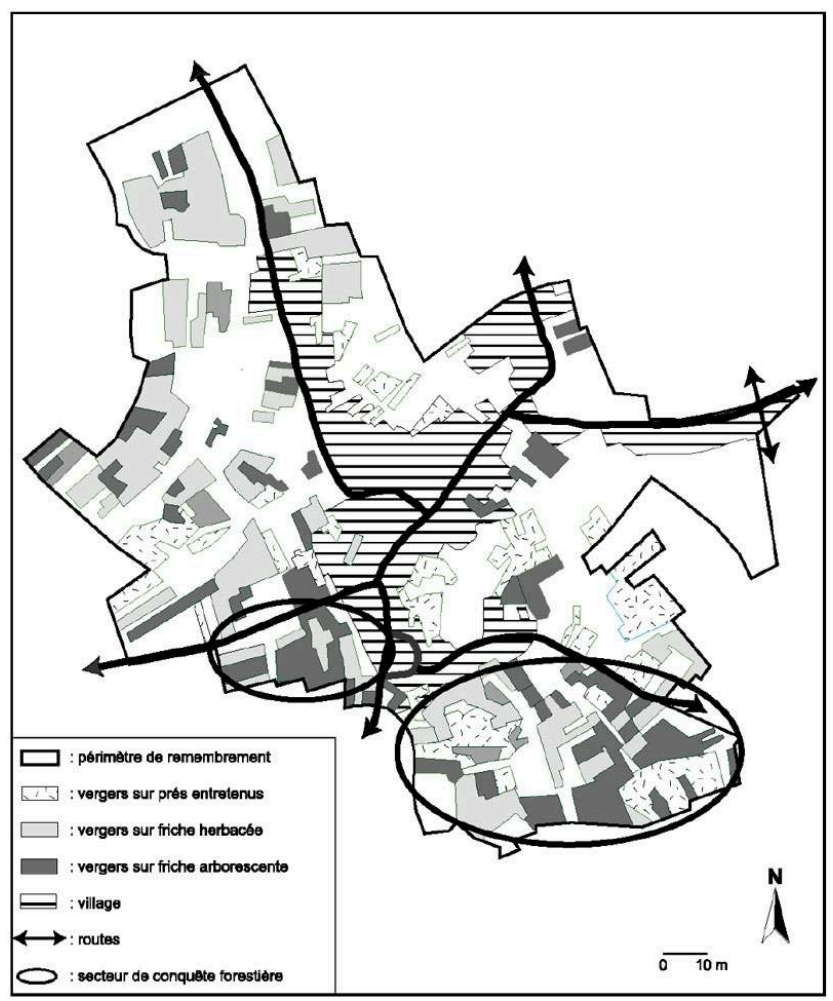

Le remembrement de Leyr a posé de nombreux problèmes et enregistré un retard de plus de dix ans. Le périmètre a connu plusieurs remaniements avant d'être divisé en deux parties, dont une concernant la zone des vergers située en périphérie du noyau villageois. Dans ce secteur, le parcellaire de vergers est plus atomisé qu'ailleurs.

d'après A. Rin, 2001

La mise en place de deux périmètres distincts avait été initialement prévue. Ce choix fut ensuite abandonné, mais les vergers sont toutefois restés traités de manière particulière en fonction de la sensibilité des secteurs. Ainsi, une emprise minimale pour le maintien des vergers périvillageois a été définie dans le cadre d'une négociation entre les agriculteurs et les habitants les plus mobilisés par cette question. Un « souspérimètre " englobe l'ensemble des vergers familiaux que les habitants souhaitent absolument maintenir. A ceci, s'ajoute une extension en zone agricole de propriété communale afin de permettre à un exploitant agricole de quitter les alentours du village quand il envisagera de s'agrandir et de se délocaliser pour répondre aux nouveaux impératifs de la loi SRU et de ceux relatifs aux installations classées. Ce mouvement concerté permet à certains propriétaires de libérer des parcelles laissées actuellement à l'état d'abandon et qui constituent de véritables verrues paysagères à l'arrière des habitations, notamment dans la partie méridionale du village.

Enfin, bien qu'il bénéficie de la même opération d'aménagement foncier que les autres sections, «l'espace verger » se différencie de «l'espace agricole » car il ne répond pas aux mêmes attentes. En effet, cette zone se caractérise par un enchevêtrement des parcelles à vocation de pâture, de culture ou de vergers utilisées pour la production familiale de fruits. Le regroupement de ce petit parcellaire n'obéit pas aux mêmes logiques que les restructurations foncières. Les propriétaires veulent conserver la structure actuelle, même si les vergers sont âgés et en mauvais état de production. La priorité ressentie réside dans la nécessité d'assurer l'accès à chaque parcelle. Dans la 
zone des vergers qui a créé tensions et polémiques, tous les terrains on été réattribués à leur propriétaire initial avec le bornage de chaque parcelle. Toute modification du parcellaire par décision de la CCAF (commission communale d'aménagement foncier) ne pourra s'opérer sans l'accord préalable des propriétaires concernés.

21 Ainsi, la commune de Leyr a-t-elle dû adapter sa politique d'aménagement rural et ses actions aux préoccupations et aux attentes de l'ensemble de sa population prête à se mobiliser pour pouvoir maintenir sa ceinture verte de vergers périvillageois. La solution de mener conjointement et en parallèle un plan paysage définissant dans ce domaine les besoins de la commune et un remembrement pouvait paraitre peu opportun et source de conflit. Cependant, l'ensemble des acteurs concernés a réussi à conduire ces opérations sans heurt, démontrant que cette solution pourra être transposée à d'autres communes. Le cas de Leyr montre qu'il y a eu une gestion articulée des biens communs dans une certaine « vision » du territoire et des biens privés (Beuret et Trechet, 2001).

\section{B. Le verger pédagogique de Maidières (Mizera, 2001)}

Commune voisine de Pont-à-Mousson, Maidières est située sur la bordure extérieure immédiate du Parc Naturel Régional de Lorraine. Le finage est soumis à la double menace de l'extension de la friche débutée à partir de la lisière forestière sommitale et du mitage de ses coteaux bien exposés par des constructions. La commune a décidé de créer un verger pédagogique afin de conserver un espace désormais jugé patrimonial. Le projet en cours de réalisation traduit une volonté locale d'intégrer les vergers dans les récentes options de politique de développement rural durable. Pour l'instant, le cas de Maidières reste expérimental. Une convention relative à la protection des vergers a été signée entre la commune et l'EPFL (Etablissement Public Foncier de Lorraine) afin de définir les engagements de chacune des parties après acquisition des terrains.

Le verger pédagogique répond à un triple objectif. Il permet de sauvegarder des parcelles de vergers traditionnels à forte valeur paysagère. Ces parcelles sont également envisagées comme un lieu d'éducation à l'environnement en zone périurbaine. Enfin, cette opération a été intégrée dans le projet pédagogique (découverte et entretien du verger) de l'école primaire de Maidières.

Ce verger de 0,53 hectares, aménagé au cours de l'automne 2001, est implanté sur les premières pentes du front de côte de Moselle, à proximité du vieux noyau villageois. Pour le protéger de toute pression foncière, il a été classé dans l'ancien POS en tant que « terrain cultivé à protéger » bien qu'il se situe en secteur urbanisé. Cette démarche de protection est fondamentale, n'aboutit pas à une inaliénabilité du terrain, mais lui confère une protection efficace contre toute velléité d'extension du lotissement voisin.

L'aménagement du verger pédagogique n'a pas nécessité de gros travaux puisqu'il était déjà composé d'arbres plutôt âgés mais relativement bien entretenus sur un sol pâturé. Les aménagements ont permis de recréer des alignements de fruitiers avec des arbres de haute tige mêlant plusieurs essences, certains des anciens arbres ayant été gardés. Un arbre mort a été conservé pour son intérêt écologique. Trois entrées ont été remises en état, de même qu'un petit muret de pierre. Compte tenu de sa situation par rapport au noyau villageois, ce verger est également envisagé comme un espace public, ludique et un lieu de promenade. Enfin, à côté des arbres fruitiers, il est prévu de créer un potager pédagogique et de planter de la vigne. 


\section{Conclusion}

Après une période d'inexorable déclin et d'enfrichement, les vergers des systèmes de côte sont désormais reconnus pour la multifonctionnalité qu'ils assurent (production économique, valeur esthétique, rôle dans la mise en scène des paysages). Façonnés par les hommes depuis plusieurs siècles et plus récemment par une nature reprenant ses droits dans le cadre d'un enfrichement généralisé, les vergers constituent une mosaïque biogéographique, reflet d'une longue évolution. Actuellement, les néo-ruraux à la recherche d'un cadre de vie de qualité sont de plus en plus nombreux à plaider en faveur du maintien de la ceinture arborée. Aménageurs et décideurs politiques ont récemment pris conscience de l'intérêt à porter à cette présence patrimoniale à préserver. Désormais, les vergers sont intégrés dans les politiques de développement rural durable. Malgré la très forte déprise qui perdure, les réflexions évoquées, les réalisations initiales abouties depuis peu montrent qu'une approche nouvelle s'impose, infléchit le mouvement en cours, en particulier dans le cadre d'application des CTE (contrats territoriaux d'exploitation ; à l'automne 2002, l'État s'engage à poursuivre la validité des contrats signés par les préfets, mais envisage de ne plus étendre l'expérience jugée coûteuse). Mener une politique de développement global des territoires inclut en Lorraine la prise en compte des vergers vivants, fonctionnels, variés, redynamisés par des opérations programmées d'amélioration.

\section{BIBLIOGRAPHIE}

BERGER A. (dir) (2001). - Dynamique de l'espace rural, environnement et stratégies spatiales, Montpellier, CNRS-Université Paul Valéry, 560 p.

BEURET J.-E., Trechet C. (2001). - Pour une gestion concertée de l'espace rural : appuyer des médiations territoriales, Paris, Courrier de I'Environnement de I'INRA, 43, pp. 5-39.

BoNNEFonT J.-C. (1974). - Les lisières forestières en Lorraine, Nancy, Revue Géographique de I'Est, 1-2, pp. 4-29.

CRoIX N. (dir.), (1998). - Environnement et nature dans les campagnes. Nouvelles politiques, nouvelles pratiques, Rennes, Presses Universitaires, 261 p.

HERVIEU B, VIARD J. (1996). - Au bonheur des campagnes : La Tour d'Aigues, DATARAube, $155 \mathrm{p}$.

Husson J.P. (2001). - Le mirabellier en Lorraine, in Territoires, paysages et héritages, mélanges offerts au Pr J.C. Bonnefont, Univ. Nancy 2, 156 p., pp. 67-70.

JACQUIN F. et al. (1994). - Sols de vignes et de vergers en Lorraine, Metz, Conseil Régional de Lorraine,123 p.

KerVAlla F. (1995). - Étude de faisabilité de l'application des mesures agrienvironnementales aux vergers de Lorraine, Metz, DIREN, 29 p. 
LECLAIRE E. (2002). - Le patrimoine vert de l'agglomération nancéienne. Espace, enjeux et outils de gestion. DEA, Univ. Nancy 2, 104 p.

MAROCHINI E (1999). - Les remembrements en Moselle entre économie, environnement et société, essai de géographie rurale appliquée, Thèse de doctorat, Univ. Metz, $599 \mathrm{p}$.

Mathy A (1998). - Les paysages du vignoble des Côtes de Toul, expression de mutations récentes, Nancy, Revue géographique de l'Est, 1-2, pp. 9-16.

Mizera J. (2001). - Dynamiques spatiales, trajectoires et paysages des vergers mussipontains, DEA Univ. Nancy 2, 70 p.

Rin A (2001). - Les vergers des Côtes de Toul, DEA Univ. Nancy 2, 88 p.

\section{RÉSUMÉS}

Situés autour des villages d'habitat groupé, les vergers étaient jusqu'à ces dernières années souvent vieillis, mal entretenus ou même détruits pour laisser place à de nouvelles constructions. Ils suscitent aujourd'hui un intérêt nouveau. Dans le cadre d'une démarche territoriale négociée, placée entre nouvelle appropriation et projet plurifonctionnel, les vergers redeviennent des zones d'enjeux. Leurs devenirs occupent une place importante dans les nouveaux débats nés de la renaissance rurale et de la nouvelle écologie des paysages.

Orchards, situated around nucleated villages, have often been, until recently, old, badly maintained or even destroyed to provide space for new developments. Today they are arousing new interest. In the context of territorial negociations about new ap- propriations and multifonctionnal plans, orchards have acquired a new significance. They occupy an important place in new debats about rural renaissance and new landscape ecologies.

Am Rande der Gruppensiedlungen gelegen, waren die Obstgärten bis in die letzten Jahre oft veraltet, schlecht gepflegt oder sogar zerstört, um Platz für neue Nutzungen zu schaffen. Sie rufen heute ein neues Interesse hervor. Im Rahmen territorialer Verhandlungen mit neuer Besitzstruktur und plurifunktionalen Vorhaben werden die Obstgärten zu Problemzonen. Ihre Zukunft nimmt einen gewichtigen Platz in den neuen Debatten ein, die aus der ländlichen Renaissance und der neuen Landschafts-ökologie entstanden sind.

\section{INDEX}

Schlüsselwörter : Ländliche Raumordnung, Lothringen, Obstgarten

Mots-clés : aménagement rural, Lorraine, verger

Keywords : Lorraine, orchard, rural development

\section{AUTEURS}

\section{AURÉLIE RIN}

BAL Conseils, 11 Grande Rue 54000 Nancy aurelie.rin@wanadoo.fr 


\section{JEAN-PIERRE HUSSON}

Université de Nancy 2 - Centre d'Études et de Recherches sur le Paysage - BP 33-97, 54015 Nancy cedex -Jean-Pierre.Husson@univ-nancy2 